EVS28

KINTEX, Korea, May 3-6, 2015

\title{
Noise emissions on switched reluctance motors: evaluation of different structural models
}

\author{
Cassio T. Faria ${ }^{1}$, Fabio Santos ${ }^{1}$, Fabien Chauvicourt $^{1}$, Stefano Orlando ${ }^{1}$ \\ ${ }^{1}$ Siemens Industry Software N.V., Interleuvenlaan 68, B-3001, Leuven (Belgium), Corresponding e-mail: \\ cassio.faria@siemens.com
}

\begin{abstract}
The mass production of electric vehicles can pressure some specific natural resources that are needed for the production of the motors utilized in such vehicles. In those lines an alternative to the use of rare-earth magnets in the propulsion system is to substitute permanent-magnet synchronous machines (PMSM) by switched reluctance motors (SRM). One of the main drawbacks of SRMs is the torque ripple and consequently the noise and vibration that it can cause in the driveline. The further development of this motor technology needs to properly understand and address the mechanical vibrations caused by the magnetic forces in the stator. The goal of this paper is to investigate the effects of increased complexity of structural modelling in capturing the physical phenomena that governs this system. By comparing the modal parameters of structures modelled by $2 \mathrm{D}$ analytical techniques, finite elements (FE) with isotropic material and FE with orthotropic properties, it is possible to discuss the limitations and applicability of each technique.
\end{abstract}

Keywords: SRM, noise, vibration, structural model, analytical, FEM.

\section{Introduction}

Green-house gases emission and consequently global warming has been a driver for the development of new vehicle propulsion technology, in particular hybrid and electrical vehicles [1]. The current market-available electric vehicles rely on the use of permanentmagnet synchronous machines (PMSM) as they have very good efficiency performances [2].

A consequence of betting on PMSM technology for a global scale conversion of the automotive fleet from combustion to electric powered engines is the need of specialized raw material for the manufacturing of permanent-magnets. These commodities are known as rare-earth materials (e.g. Samarium, Neodymium) and the pressure introduced on such by pushing for a massscale production could significantly impact the final price of this new technology and also create conflict over those resources [3].

A solution is to invest efforts in the development of permanent-magnet (PM) free motors. In this line of thought switched reluctance machines (SRM) presents themselves as a great alternative as the production of torque through reluctance eliminates the need for PM [4]. Figure 1 illustrates the simple mechanical construction of a SRM. At these machines the complexity lies within the electrical design as the current needs to be switched from each phase with precise timing. 


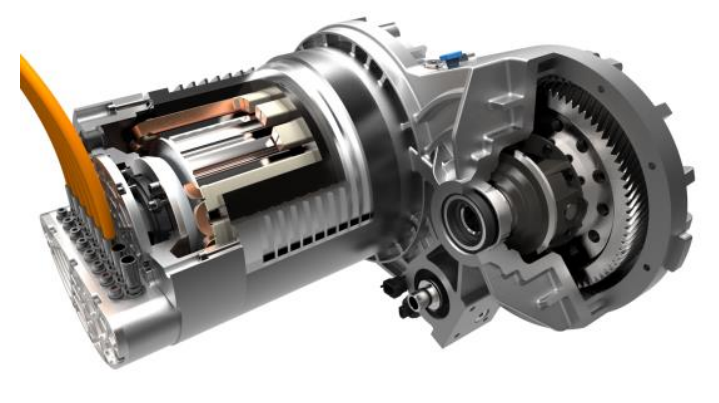

Figure 1: SRM motor (on the left) with integrated gearbox, courtesy of Punch Powertrain

One of the main issues associated with SRM is the acoustic noise emission as the torque ripple can very easily excite structural modes of the motor and maximize the transfer of mechanical energy to the surrounding air [5]. It becomes crucial to appropriately include in the motor design process measures to reduce the acoustic emissions of the motor and improve its noise, vibration and harshness (NVH) behaviour [6].

One of the key aspects while modelling the mechanical structure of a SRM is to appropriately capture the dynamics of the main structural component, i.e. stator core. Since this component is manufactured through the stacking of steel laminas, in order to reduce iron losses in the stator, several structural nonlinearities are introduced to the system (e.g. friction between layers, residual stress/strain in the laminas, etc) [7].

The goal of this paper is not to compose a structural model that adequately captures all these nonlinear effects, but rather explore the prediction capabilities of simpler models as they can be very useful for the design and prediction of overall trends of the motor.

In section 2 the analytical model of the stator is explored, followed by a discussion on finite element models (FEM) using LMS Virtual.Lab® in section 3. Section 4 presents the description of a simple $12 / 8$ SRM stator with the calculations of modal properties (natural frequencies and mode shapes) for the three modelling approaches: analytical; FEM with isotropic element properties; FEM with orthotropic properties. The results are discussed and conclusions are drawn in the last section.

\section{Analytical structural model}

Considering a ring of density $\rho$, Young's modulus $E$, second moment of area $I$, area of cross-section $A$ and radius of curvature $R$ as illustrated in Figure 2 below.

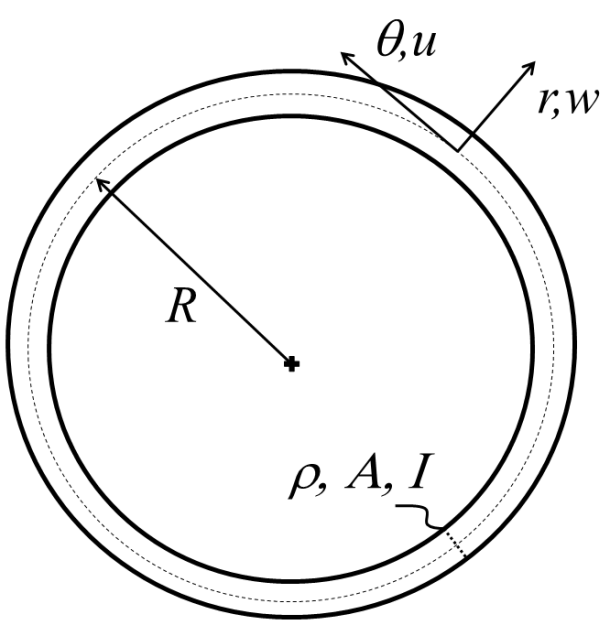

Figure 2: Circular ring vibrating in its plane ( $\theta$ and $r$ directions)

One can easily compose a free-body diagram and establish the equilibrium equations. After neglecting the rotational inertia of the differential element (ordinary beam theory - Euler-Bernoulli) one can obtain [8]:

$$
\begin{aligned}
& u_{t t}+\alpha A\left(w_{\theta}+u_{\theta \theta}\right)-\alpha \beta\left(u_{\theta \theta}-w_{\theta \theta \theta \theta}\right)=0 \\
& w_{t t}+\alpha A\left(w+u_{\theta}\right)-\alpha \beta\left(u_{\theta \theta \theta}-w_{\theta \theta \theta \theta}\right)=0
\end{aligned}
$$

where subscript $t$ indicates a derivative with respect to time and subscript $\theta$ with respect to the angle. Also:

$$
\begin{aligned}
& \alpha=\frac{E}{\rho A R^{2}} \\
& \beta=\frac{I}{R^{2}}
\end{aligned}
$$

In Eq. (1) the upper equation represents the circumferential equilibrium and the lower one the radial.

For a free-floating circle/ring the boundary condition is the periodicity, which means:

$u(\theta+2 \pi, t)=u(\theta, t)$

$w(\theta+2 \pi, t)=w(\theta, t)$

Assuming the following form to the solution:

$u(\theta, t)=U e^{i(n \theta-\omega t)}$

$w(\theta, t)=W e^{i(n \theta-\omega t)}$

where $U$ and $W$ are constants and $n$ is an integer values ranging from 0 to infinity.

One can obtain the following characteristic equation which relates the natural frequency to the wave number:

$\omega^{4}-\alpha \omega^{2}\left(A+\beta n^{2}\right)\left(1+n^{2}\right)+\alpha^{2} n^{2} A \beta\left(1-n^{2}\right)^{2}=0$ 
Each solution pair of Eq. (5) can be plugged back into Eq. (4) and consequently to Eq. (1) in order to find the constant values $\mathrm{U}$ and $\mathrm{W}$ that will form the mode shapes.

At this point it should be obvious that this formulation does not capture the true form of a motor stator, which is a circle with indentations, making the physical properties used in the model $\theta$ dependent, as illustrated by figure 3 .

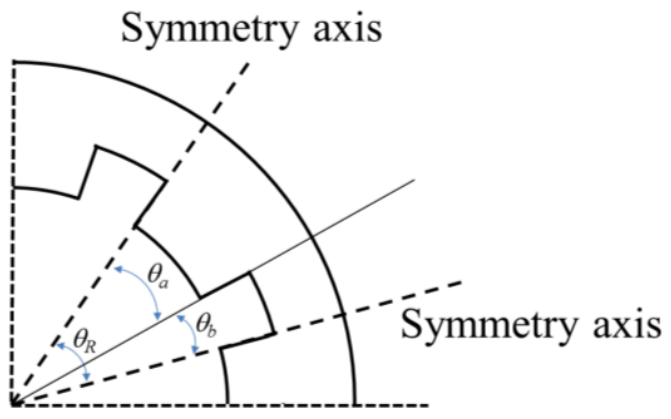

Figure 3: Detail of a stator structure section

The solution implemented here is to modify the ring properties by considering that the structure is a series of identic structures connected to its neighbour. By implementing a homogenization method of angle averaging one can define the new physical properties to be input into Eq. (5):

$P=\frac{P_{a} \theta_{a}+P_{b} \theta_{b}}{\theta_{R}}$

where in this case $\mathrm{P}$ represents any of the properties input in Eq. (5), i.e. $R, A, \rho$.

The subscript $a$ and $b$ represent the regions where those properties are homogeneous inside each symmetric section of the stator, as illustrated by figure 3 .

It is important to note that only the second moment of area (I) is not homogenized as this would cause an unrealistic increase in structural stiffness due to the cubic term used in its calculation.

\section{$3 \quad$ FEM in LMS Virtual.Lab®}

Finite element models are in its essence a formal procedure to spatially discretize a structural domain and to attribute to each local unit/element a type of solution that is proportional to a few neighbouring points, referred to as nodes. A global solution is obtained by determining or characterizing the behaviour of this finite number of points.

In practical terms the procedure can be simplified to the following steps:
1. Geometry definition;

2. Nodes and elements definition (meshing);

3. Choose types and properties for the elements;

4. Assembly of the global problem matrix

5. Solution of a linear system

LMS Virtual.Lab® is a software tool developed by Siemens Industry Software that is not only capable of handling the full process of a FEM simulation but goes beyond by offering a complete set of solutions for 3D simulations [9].

Inside the LMS Virtual.Lab ${ }^{\circledR}$ environment the first step of the FEM process can be accomplished by either sketching the desired geometry or by importing the CAD drawing. Several tools are also available to automatically create and edit the mesh, libraries of commonly used materials and several types of elements are available to be chosen from. In other words, the software takes care of the FEM process with a user-friendly interface.

Of particular interest for the analysis carried in this paper is the definition of two different element types: the isotropic and orthotropic 3D material properties. The stress-strain relationship considered is linear and is written as:

$\left[\begin{array}{l}\varepsilon_{11} \\ \varepsilon_{22} \\ \varepsilon_{33} \\ \gamma_{23} \\ \gamma_{31} \\ \gamma_{12}\end{array}\right]=\mathbf{S}_{6 \times 6}\left[\begin{array}{l}\sigma_{11} \\ \sigma_{22} \\ \sigma_{33} \\ \sigma_{23} \\ \sigma_{31} \\ \sigma_{12}\end{array}\right]$

where $\mathrm{S}$ is the compliance matrix, particularly defined for each type of material, $\varepsilon$ is the engineering strain, $\gamma$ is the engineering shear strain, $\sigma$ the stress and the subscripts indicate material lattice directions.

For isotropic material the compliance matrix takes the following form [10]:

$$
\mathbf{S}_{i s o}=\left[\begin{array}{cccccc}
\frac{1}{E} & \frac{-v}{E} & \frac{-v}{E} & 0 & 0 & 0 \\
\frac{-v}{E} & \frac{1}{E} & \frac{-v}{E} & 0 & 0 & 0 \\
\frac{-v}{E} & \frac{-v}{E} & \frac{1}{E} & 0 & 0 & 0 \\
0 & 0 & 0 & \frac{2(1+v)}{E} & 0 & 0 \\
0 & 0 & 0 & 0 & \frac{2(1+v)}{E} & 0 \\
0 & 0 & 0 & 0 & 0 & \frac{2(1+v)}{E}
\end{array}\right]
$$

where $v$ is the Poisson's ratio.

The symmetry of the compliance matrix is clearly stated (the matrix is identical to its transpose) and such property reflect the isotropic characteristic of the material.

Meanwhile, the relationship between stress and strain for an orthotropic material is somehow 
different. The symmetry property does not hold and for the particular case it is considered a planar symmetry (same compliance coefficients for directions 1 and 2):

$\mathbf{S}_{\text {ortho }}=\left[\begin{array}{cccccc}\frac{1}{E_{1}} & \frac{-v}{E_{1}} & \frac{-v}{E_{3}} & 0 & 0 & 0 \\ \frac{-v}{E_{1}} & \frac{1}{E_{1}} & \frac{-v}{E_{3}} & 0 & 0 & 0 \\ \frac{-v}{E_{1}} & \frac{-v}{E_{1}} & \frac{1}{E_{3}} & 0 & 0 & 0 \\ 0 & 0 & 0 & \frac{1}{G_{23}} & 0 & 0 \\ 0 & 0 & 0 & 0 & \frac{1}{G_{31}} & 0 \\ 0 & 0 & 0 & 0 & 0 & \frac{1}{G_{12}}\end{array}\right](9)$

where $G$ is the shear modulus.

\section{Structural model and results}

The 12/8 SRM motor utilized in this study was designed by the software JMAG ${ }^{\circledR}$ based on the specifications described by [11]. The constraints given to the software are presented in table 1 and the resulting stator geometry designed by the software is exported to LMS Virtual.Lab®.

Table 1: Values used in the design of a 12/8 SRM motor in JMAG®

\begin{tabular}{|c|c|}
\hline Peak power & $30 \mathrm{~kW}$ \\
\hline Peak torque & $200 \mathrm{~N} / \mathrm{m}$ \\
\hline Speed range & $0-10000 \mathrm{rpm}$ \\
\hline Voltage supply & $307 \mathrm{~V}$ \\
\hline Max outer diameter & $220 \mathrm{~mm}$ \\
\hline Max length & $275 \mathrm{~mm}$ \\
\hline Number of slots/poles & $12 / 8$ \\
\hline
\end{tabular}

Now that the geometry of the stator is known, it can be used to evaluate the different structural models described by this paper. At all study cases, steel is taken as the constituent material, which implies in the following parameters: Young's modulus - 2e11 N/m2; Poison's ratio 0.266 ; and density of $7860 \mathrm{~kg} / \mathrm{m} 3$.

For the orthotropic case, the Young's modulus for the longitudinal (3) direction is reduced by $10 \%$ to emulate the stiffness reduction due to the stacking of the different lamina. Also, the shear modulus for directions 23 and 31 are chosen to be $20 \%$ of the steel Young's modulus, while for the direction 12 it is $40 \%$. The big discrepancy is to emulate the friction (slipping/relative motion) between laminas. The other properties are kept the same.

\subsection{Analytical results}

Figure 4 below illustrates the final motor topology and also describes the most significant dimensions of the motor. It is important to mention that the coil diameter is the ring diameter at the root of the tooth and it is the dimension used for the homogenization process.

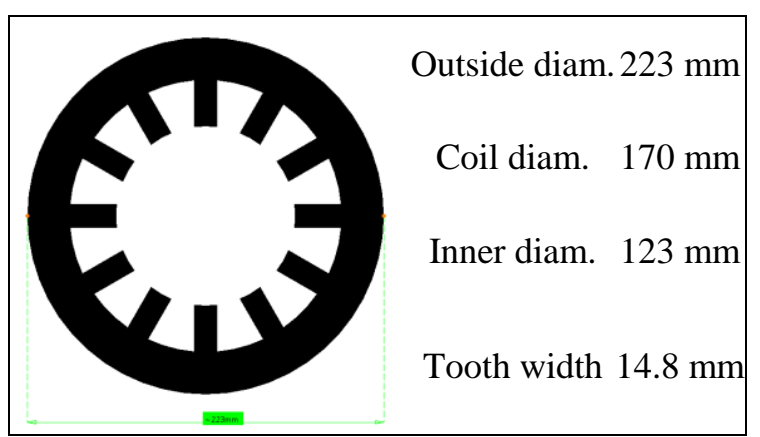

Figure 4: Stator geometry description

At this stage, equation (5) can be used to calculate the natural frequencies of the structure, more specifically, for each wave number one should have 4 roots of that particular polynomial equation. Because the equation has no odd coefficients it is also expected that only two of those roots are unique, with the other two being its conjugated. Physically this means that each mode-shape that occurs at a particular frequency have a twin pair that occur 90 degrees apart.

The mode shapes and natural frequencies of the stator under investigation using the analytical approach are given by figure 5 . It is important to mention that the rigid body modes $(\omega=0)$, that occur for wave number zero and one, are not depicted.

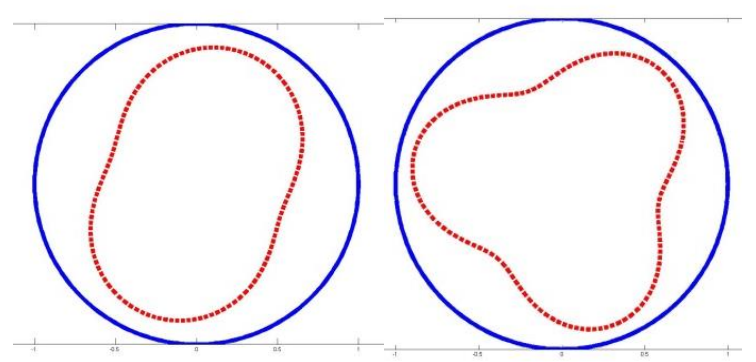
(a) $2-1635 \mathrm{~Hz}$
(b) $3-4617 \mathrm{~Hz}$ 


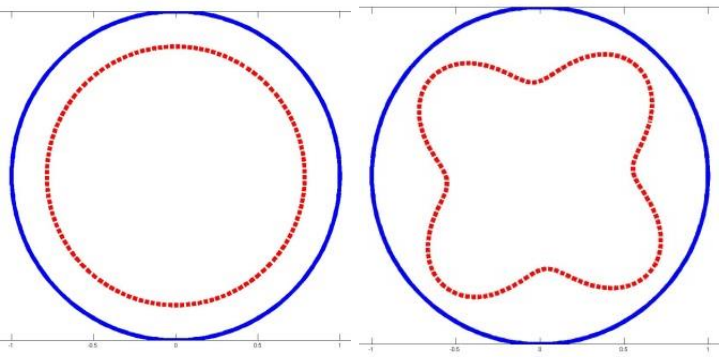

(c) $0-8609 \mathrm{~Hz}$

(d) $4-8842 \mathrm{~Hz}$

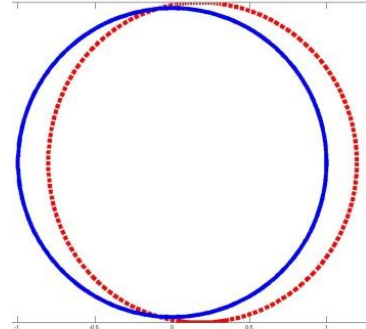

(e) $1-12206 \mathrm{~Hz}$

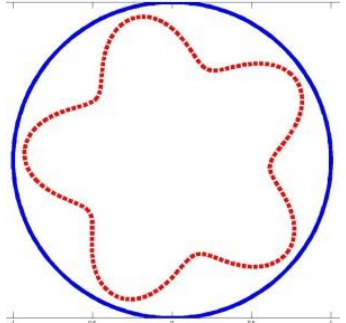

(f) $5-14287 \mathrm{~Hz}$
Figure 5: Mode shapes for analytical formulation; the first number on the individual caption is the wave number $(n)$ and the second is the natural frequency converted to $\mathrm{Hz}$

It is common to see reference in literature to names for the modes with particular wave numbers, for example, figure $5(\mathrm{a})$ is called the ovalization mode, while $5(\mathrm{c})$ is called breathing mode. Other names are also common, such as: triangular mode 5(b), square mode 5(d) and so on.

\subsection{FEM isotropic results}

The transfer of the geometry produced by the motor design process within JMAG® to the LMS Virtual.Lab is a fairly simple process that accelerates the modal evaluation of the SRM motor.

Starting from the geometry a mesh is created to represent the structure. This cloud of points (nodes) is interconnected by defining a relation for the volume delimited by a small subgroup of nodes, called element. For this problem a PENTA6 element was selected, which implies that the volumes are triangular prism with five faces and defined by six nodes.

The resulting mode shapes of the analysis carried out considering solid 3D isotropic material properties to the stator are presented by Figure 6 . The resulting natural frequencies are also given in the caption of each individual mode shape. The search for mode shapes within LMS Virtual.Lab was limited to $5000 \mathrm{~Hz}$.

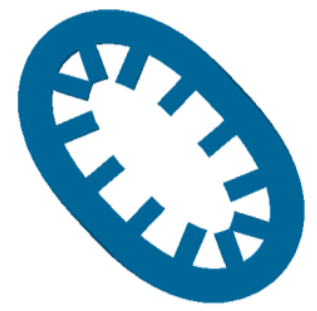

(a) $-1566 \mathrm{~Hz}$

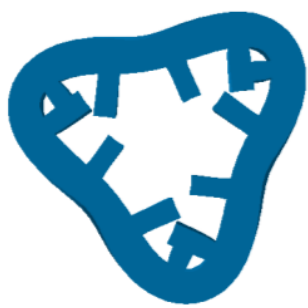

(c) $-3991 \mathrm{~Hz}$

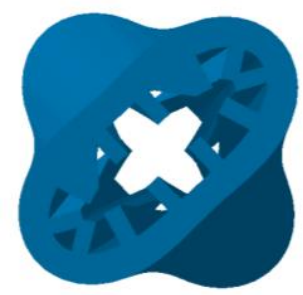

(b) $-1875 \mathrm{~Hz}$

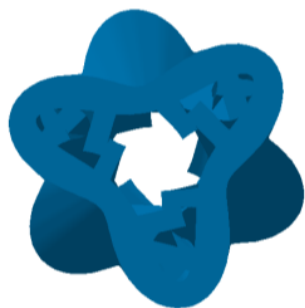

(d) $-4381 \mathrm{~Hz}$

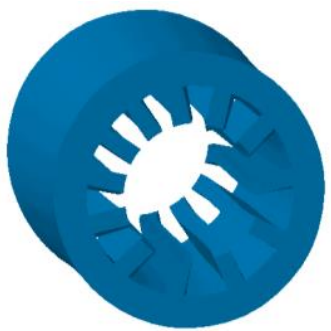

(e) $-4964 \mathrm{~Hz}$

Figure 6: Mode shapes for isotropic FEM analysis (symmetric modes omitted)

When compared to the analytical solution it is clear that modes that contain an axial component are present, i.e. 6(b) and 6(d). This out of plane motion is not captured by the planar formulation considered in this paper. The same rationale applies to the axial bending mode, figure 6(e).

\subsection{FEM orthotropic results}

By repeating the $\mathrm{FE}$ model creation process described in the previous subsection but making the use of $3 \mathrm{D}$ orthotropic material properties, one can obtain the modal parameters of the model. To capture the dynamic effects of the laminated stator the materials properties are adjusted in particular directions of the stator.

The Young's modulus in the axial direction is reduced to capture the imperfect stacking of the laminas. Meanwhile, the shear modulus is also reduced in the 23 and 31 directions to emulate the slipping (friction) between layers. The results of such simulation are presented in figure 7 . The search for mode shapes within LMS Virtual.Lab is limited to $5000 \mathrm{~Hz}$. 


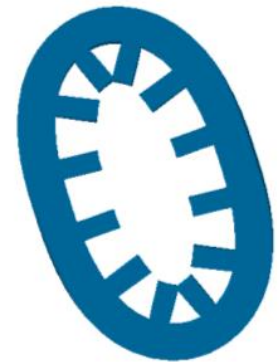

(a) $-1563 \mathrm{~Hz}$

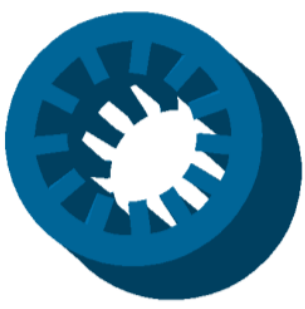

(c) $-3712 \mathrm{~Hz}$

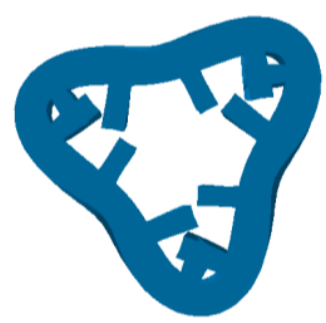

(e) $-3981 \mathrm{~Hz}$

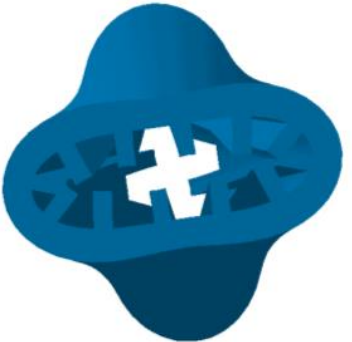

(b) $-1705 \mathrm{~Hz}$

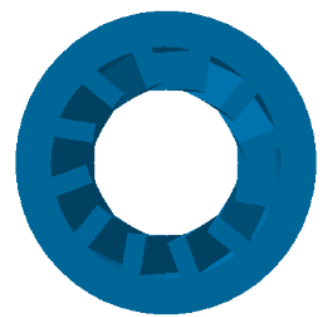

(d) $-3838 \mathrm{~Hz}$

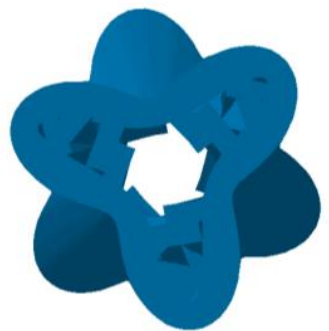

(f) $-4158 \mathrm{~Hz}$

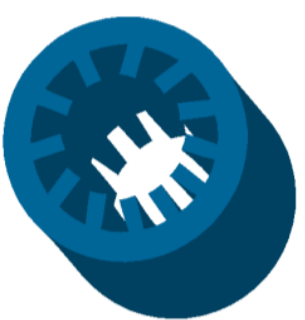

(g) $-4725 \mathrm{~Hz}$

Figure 7: Mode shapes for orthotropic FEM analysis (symmetric modes omitted)

The fact that the properties are the same in the planar direction compared to the isotropic case, results in the planar mode shapes and natural frequencies (Figure 7a and 7e) to match the ones found using isotropic properties (6a and $6 c$ ). However the reduction of stiffness in the axial direction anticipates the occurrence of axially asymmetric modes (figures $7 \mathrm{~b}$ and $7 \mathrm{f}$ ) when compared the simulation using isotropic material properties (figure $6 \mathrm{~b}$ and $6 \mathrm{~d}$ ).

The appearance of a torsional mode, figure $7 \mathrm{~d}$, is due to the lower shear modulus in the 23 and 31 directions. This mode is not present within the investigated frequency range for the isotropic simulations.

\section{Discussion and conclusion}

The analytical modelling approach presented on section 2 is a fast and powerful approach to predict modal parameters of a stator structure. Moreover, it provides important physical insight into the dynamic problem at hand.

The polynomial form of equation (5) implies in the existence of four natural frequency values for each wave number. The fact that such equation has no odd coefficients also means that only two of those frequencies are unique. The non-unique eigenvalues will produce the symmetric mode shapes, i.e. eigenvector shifted 90 degrees.

The wave number results in the number of dents/notches that can be seen on the mode shapes as can be seen on figure (5). It is important to mention that $n$ values of zero and one also produce rigid body modes, i.e. zero natural frequency.

The homogenization technique proposed for the properties input in the model does not fully capture the changes induced by the presence of the teeth in the structure but is a first simple step to take it into account. By comparing the prediction of the natural frequency for the ovalization mode, for example, the analytical model (figure 5a) has a higher value when compared to the FEM models (figures 6a and 7a). This fact indicates that either the structural stiffness is overestimated in the analytical model or the mass of the teeth are not properly accounted for.

On the other end of the simulation spectrum the finite element solution is a much more expensive and time consuming prediction tool. However, the results are more representative of the physical system as much of its geometric characteristics are captured by the process. When considering isotropic material properties for the stator structure not only the in-plane mode shapes are found but also the deformations along axial direction, such as the ones displayed in figures (6b), (6d) and (6e).

When comparing both FEM solutions, using isotropic and orthotropic material properties, it is possible to see the effect of particular coefficients. For instance, a reduction in the axial Young's modulus anticipates the occurrence of axially asymmetric modes, such as (7b) and (7f) when compared to $(6 \mathrm{~b})$ and $(6 \mathrm{~d})$. The reduced shear modulus on the 12 direction (planar) also has a very characteristic effect of anticipating the pure torsional mode (figure 7d).

These characteristic effects of the material property in one particular direction can be the basis of an efficient model updating technique to allow the FEM simulations using orthotropic properties to match the experimental results. By observing 
the experimental mode shapes (or deflection shapes) one can tune the model parameters to position these modes relative to one another.

In summary, this paper presented and compared three different modelling techniques with increased complexity, i.e. analytical, FEM with isotropic material and FEM with orthotropic. These approaches were used to provide physical insight in to the problem at hand and also can allow for increased prediction capabilities of the model.

\section{Acknowledgments}

The authors would like to acknowledge the financial support of the European Commission via the FP7 - Transport - ARMEVA project (605195). It is also important to recognize JSOL Corporation for the JMAG ${ }^{\circledR}$ licenses made available to the authors and Punch Powertrain for the valuable input during this process.

\section{References}

[1] J.H. Williams, A. DeBenedictis, R. Ghanadan, A. Mahone, J. Moore, W.R. Morrow, S. Price, and M.S. Torn, The Technology Path to Deep Greenhouse Gas Emissions Cuts by 2050: The Pivotal Role of Electricity, Science 335, 6064(2012), 53-59.

[2] X. Yanliang, X. Jiaqun, W. Wenbin, and T. Renyuan, Development of Permanent Magnet Synchronous Motor Used in Electric Vehicle, Proceedings of the Fifth International Conference on In Electrical Machines and Systems ICEMS, 2(2001), 884-87.

[3] M Humphries Rare Earth Elements: The Global Supply Chain. DIANE Publishing Company, 2010.

[4] R. Krishnan, Switched Reluctance Motor Drives: Modeling, Simulation, Analysis, Design, and Applications, CRC Press Industrial Electronics., 2001.

[5] F.L.M. Dos Santos, J. Anthonis, F. Naclerio, J.J.C. Gyselinck, H. Van der Auweraer, and L.C.S. Goes, Multiphysics NVH Modeling: Simulation of a Switched Reluctance Motor for an Electric Vehicle, IEEE Transactions on Industrial Electronics, 61-1(2014), 469-76.

[6] H. Van der Auweraer and K Janssens, A SourceTransfer-Receiver Approach to NVH Engineering of Hybrid/Electric Vehicles, SAE Technical Paper 2012-36-0646 (2012).

[7] P. Miha, G. Čepon, and M. Boltežar, Introduction of the Linear Contact Model in the Dynamic Model of Laminated Structure
Dynamics: An Experimental and Numerical Identification, Mechanism and Machine Theory 64-0(2013), 144-54.

[8] A. Dasgupta, "Lecture notes of Vibration of Structures: lesson 29, Dynamic of curved beams, http://nptel.ac.in/courses/112105054/, accessed on 2015-01-13.

[9] Noise and Vibration: LMS Virtual.Lab brochure, http://www.plm.automation.siemens.com/en_us/pr oducts/lms/virtual-lab/nvh/index.shtml, accessed on 2015-01-10.

[10] A.P. Boresi, Advanced Mechanics of Materials, 6th ed. John Wiley \& Sons Canada, Limited (2004).

[11] S. Faid, P. Debal, and S. Bervoets, Development of a Switched Reluctance Motor for Automotive Traction Applications, EVS25Electric Vehicle Symposium (2010).

\section{Authors}

\begin{tabular}{|l|l|} 
Cassio Faria is a Sr. Project Engineer \\
for R\&D at Siemens Industry \\
Software NV and his work focus on \\
NVH of electric machines, design of \\
smart structures, nonlinear controller \\
design and virtual sensing techniques. \\
He earned his PhD in Mechanical \\
Engineering from the Virginia \\
Polytech Institute and State University \\
Virginia Tech) in 2013 and later did \\
his Postdoc at the Aerospace \\
Engineering department in the \\
University of Michigan. \\
\hline $\begin{array}{l}\text { Fábio L. M. dos Santos was born in } \\
\text { Rio Claro, Brazil, in 1985. He } \\
\text { received the B.Sc. degree in control } \\
\text { and automation engineering from the } \\
\text { University of Campinas, Campinas, } \\
\text { Brazil, and the M.Sc. degree from the } \\
\text { Politecnico di Torino, Turin, Italy, in } \\
\text { 2008. He is currently a Research } \\
\text { Engineer with Siemens Simulation \& } \\
\text { Test Solutions, Leuven, Belgium, } \\
\text { while also pursuing a joint Ph.D. } \\
\text { degree at the Instituto Tecnológico de } \\
\text { Aeronáutica, in São José dos Campos, } \\
\text { Brazil and at the Katholike } \\
\text { Universiteit Leuven, in Leuven, } \\
\text { Belgium. } \\
\text { Fabien Chauvicourt was born in } \\
\text { Poissy, France, in 1991. He received } \\
\text { the M.Sc. degree from the Université } \\
\text { de Technologie de Compiègne, } \\
\text { Compiègne, France, in 2014. } \\
\text { He is currently a Research Engineer } \\
\text { within Siemens Industry Software } \\
\text { NV, Leuven, Belgium, as a Fellow of } \\
\text { the ADEPT Project, which is a }\end{array}$ \\
\hline
\end{tabular}




\begin{tabular}{|l|l|}
\hline & $\begin{array}{l}\text { European Commission ITN Marie } \\
\text { Curie Programme, and he is also a } \\
\text { Ph.D. Researcher at the Katholieke } \\
\text { Universiteit Leuven, Leuven, Belgium } \\
\text { and the Université Libre de Bruxelles, } \\
\text { Bruxelles, Belgium. }\end{array}$ \\
\hline $\begin{array}{l}\text { Stefano Orlando obtained his Master } \\
\text { Science degree in mechanical } \\
\text { engineering from Universita' } \\
\text { Politecnica delle Marche (Italy) in } \\
\text { 2010. He ever since joined LMS } \\
\text { Engineering (Siemens Industry } \\
\text { Software) where he works as project } \\
\text { leader, taking care of the management } \\
\text { and execution of noise and vibration's } \\
\text { related project in the automotive and } \\
\text { aerospace sector. }\end{array}$ \\
\hline
\end{tabular}

